\title{
Multi-collector Inductively Coupled Plasma Mass Spectrometry: New Developments and Basic Concepts for High-precision Measurements of Mass- dependent Isotope Signatures
}

\author{
Nicolas D. Greber ${ }^{\star a b}$ and Kirsten van Zuilen ${ }^{c}$
}

\begin{abstract}
Due to the development of multi-collector inductively coupled plasma mass spectrometry (MC-ICPMS) around 25 years ago, the isotopic compositions of a large range of elements (masses from Li to $U$ ) are now analyzed with high enough precision and accuracy to resolve subtle natural variations. These so-called 'nontraditional stable isotope systems' opened many new research avenues and are applied at an increasing rate in industry and research projects and in a broad range of different disciplines, including archeology, biology, physics, cosmochemistry and geology. Here, we briefly summarize the most basic concepts of MC-ICP-MS, introduce new technical developments and address important points on how to acquire accurate high-precision isotope measurements of non-traditional stable isotopes.
\end{abstract}

Keywords: MC-ICP-MS · Non-traditional stable isotopes

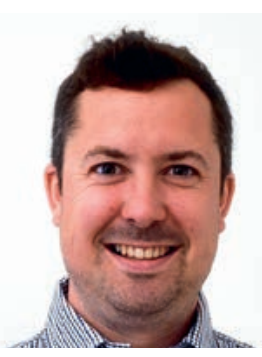

Nicolas Greber is an isotope geologist working at the Natural History Museum of Geneva, Switzerland. He studied and finished his $\mathrm{PhD}$ in Earth Sciences at the University of Bern $(\mathrm{CH})$ in 2014. After his $\mathrm{PhD}$, Nicolas worked as a Postdoc at the University of Chicago (USA) and the University of Geneva $(\mathrm{CH})$ and as Assistant Professor at the University of Bern $(\mathrm{CH})$. His research uses variations in isotope ratios to look at magmatic processes and their impact on the biosphere in deep time. For this work, his analytical focus lies on high-precision measurements of isotopic compositions with multiple-collector inductively coupled plasma mass spectrometry.

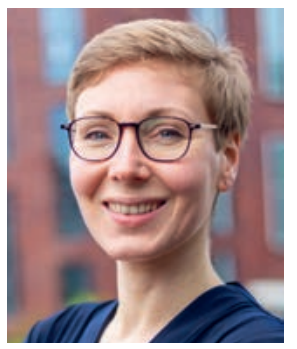

Kirsten van Zuilen studied Earth Sciences at Leibniz University of Hannover, Germany and obtained her $\mathrm{PhD}$ in Geochemistry at the University of Bergen, Norway. Since being a student assistant, she has been analyzing and investigating non-traditional isotopes in a variety of geological materials. After post doc positions held in Bern, Switzerland and Paris, France, she is now lab manager of the element analysis and mass spectrometry laboratory at the Vrije Universiteit Amsterdam, The Netherlands, where she is responsible for the clean room laboratory and two mass spectrometers. She particularly enjoys teaching isotope analysis to students and fellow researchers.

\section{Introduction}

The measurement and application of isotope variations has a long-standing tradition in the natural sciences, ranging from tracing radioactive material, dating archeological artifacts, investigating biological metabolism, looking at atmosphere processes, determining the origin of elements in solar objects and dating geological events like large magmatic eruptions, Earth's core formation or the extraction of continental crust from Earth's mantle. ${ }^{[1-6]}$ Variations in the isotope abundances of a substance, for instance, can be produced by radioactive decay $\left(e . g .{ }^{235} \mathrm{U}\right.$ and ${ }^{238} \mathrm{U}$ decay to ${ }^{207} \mathrm{~Pb}$ and ${ }^{206} \mathrm{~Pb}$ ), the production of isotopes by highenergy cosmic rays (e.g. ${ }^{10} \mathrm{Be}$ production by cosmic ray spallation of nitrogen and oxygen) or mixing of isotopically distinct regions in the solar system (e.g. nucleosynthetic anomalies of ${ }^{92} \mathrm{Mo}$ in meteorites). Chemical or physical processes are further able to fractionate isotopes of an element, i.e. different isotopes of that element partition to different degrees in coexisting phases in a natural system. The difference in the partitioning behavior can be related to the mass of the isotopes (mass-dependent fractionation) or it can be mass-independent. Mass-independent isotope fractionation is uncommon on the Earth's surface today. It is usually caused by photochemical or spin-forbidden reactions (e.g. mass-independent $\mathrm{S}$ and $\mathrm{Hg}$ isotope fractionation due to photochemical reactions in the atmosphere[7,8]).

Most isotope fractionation observed in natural samples, however, is governed by mass-dependent processes. ${ }^{[9]}$ Here, the mass of an atom affects the force constants of chemical bonds as well as the vibrational, rotational and translational energy of atoms. ${ }^{[10]}$ The small difference in mass between isotopes of an element is sufficient to influence their stability and behavior during physicochemical processes. Virtually all physicochemical 
processes, from evaporation-condensation processes in the water cycle, over metabolic processes in plants and animals to the differentiation of a planetary body into an $\mathrm{Fe}-\mathrm{Ni}$ core and a silicate mantle leave behind fingerprints in the form of a mass-dependent isotopic signatures.

As it is difficult to determine absolute isotope abundances of an element in a sample to a high precision and accuracy, isotopes are always measured and reported as ratios. For massdependent fractionation, the observed isotopic ratios scale with the difference in mass of the isotopes involved. For example, the fractionation of ${ }^{18} \mathrm{O}$ relative to ${ }^{16} \mathrm{O}$ is around 2 times larger than between ${ }^{17} \mathrm{O}$ and ${ }^{16} \mathrm{O}$. However, changes in the isotope ratios ${ }^{18} \mathrm{O} /{ }^{16} \mathrm{O}$ and ${ }^{17} \mathrm{O} /{ }^{16} \mathrm{O}$ are small, usually less than $5 \%$. For elements with heavier masses these changes are often even smaller than $0.1 \%$. Therefore, isotope ratios are reported relative to a common reference material using the $\delta$-notation defined as:

$$
\delta^{\mathrm{j} / \mathrm{i}} \mathrm{A}(\% 0)=\left(\frac{{ }^{\mathrm{j} / \mathrm{i}} \mathrm{A}_{\text {Sample }}}{{ }^{\mathrm{j} / \mathrm{i}} \mathrm{A}_{\text {Reference Material }}}-1\right) \cdot 1000
$$

where $j$ and $i$ are two different isotopes of element $A$. A further advantage of the $\delta$-notation is that it enables different laboratories to compare their results. For mass-dependent isotope fraction it follows that two different $\delta$-values of the same element follow a linear correlation with the slope defined by the difference of the masses of the isotopes used; for example, $\delta^{18} /{ }^{16} \mathrm{O}$ and $\delta^{17} /{ }^{16} \mathrm{O}$ are linearly correlated by $\delta^{18} /{ }^{16} \mathrm{O} \approx 2 \times \delta^{17} /{ }^{16} \mathrm{O}$. Because the relative mass difference between isotopes of light elements is bigger than of heavy elements, the degree of mass-dependent isotope fractionation is strongest among light elements and thus analytically easier to detect. Instruments capable of precisely determining isotope ratios of $\mathrm{H}, \mathrm{C}, \mathrm{O}, \mathrm{N}, \mathrm{S}$, and $\pm \mathrm{Si}$ were established in the natural sciences already around 1950. ${ }^{[11,12]}$ However, analytical development and improvement on sample preparation over the last 20 years made it possible to measure the isotopic composition of many other elements to a sufficiently high precision and accuracy to be able to resolve subtle isotope fractionation.

A key development was the construction of a multi-collector inductively coupled plasma mass spectrometer (MC-ICP-MS), an instrument that combines a magnetic sector field mass spectrometer with multiple collectors that allow to analyze several isotopes simultaneously. Furthermore, the plasma source makes the instrument very versatile, as elements with an ionization potential of below $10 \mathrm{eV}$ are ionized with an efficiency of above $60 \% .{ }^{[13,14]}$ Therefore, one can nowadays measure the isotopic composition of a large range of elements at low concentrations. Currently, measurement protocols are published for the analysis of the isotopic composition of $\mathrm{Mg}$, $\mathrm{Ca}, \mathrm{Ti}, \mathrm{V}, \mathrm{Cr}, \mathrm{Fe}, \mathrm{Ni}, \mathrm{Cu}, \mathrm{Zn}, \mathrm{Ga}, \mathrm{Se}, \mathrm{Rb}, \mathrm{Sr}, \mathrm{Zr}, \mathrm{Mo}, \mathrm{Ru}, \mathrm{Sn}$, $\mathrm{Te}, \mathrm{Ba}, \mathrm{W}, \mathrm{Hg}, \mathrm{Tl}$ and $\mathrm{U}$, and for many of these systems the isotopic compositions can be determined with an intermediate measurement precision of $\leq \pm 0.03 \%$ o per atomic mass unit (2SD) when using a MC-ICP-MS (for $\delta$-values, not absolute abundances of isotopes). Therefore, the new developments in mass spectrometry and sample preparation are offering the science community an incredible toolbox (often summarized as non-traditional stable isotopes) to approach many scientific questions from a new angle.

High-precision analysis of non-traditional stable isotopes is not trivial. Here, we summarize some of the most important concepts on how to perform high-precision isotope measurements in solution mode using a MC-ICP-MS.

\section{Instrumentation Basics}

In the last years, many excellent manuscripts have been written which describe the concepts, principles and designs of MC-ICP-MS. We therefore provide only a short summary of the most important functionalities of a MC-ICP-MS and we forward the interested readers to articles that discuss the built up of these instruments in more detail[13-16] and that have been used to write the short summary below.

Multi-collector inductively coupled plasma mass spectrometers are instruments that combine an ICP plasma source, an energy filter, a magnetic sector analyzer and several collectors that allow the simultaneous measurement of different isotopes (Fig. 1). The sample material is introduced into the plasma as an aerosol (gas or vapor). Most often, this is done by either converting a solution into a vapor with a nebulizer system, or a solid into a gas with a laser ablation system. In the plasma, the elements are ionized at temperatures of about 6000 to $8000 \mathrm{~K}$. The ions are then extracted from the plasma into the mass spectrometer in the interface and ion optics regions. Here, instrument designers face several challenges; a) the ions need to be sampled, b) the atmospheric pressure in the ICP source needs to be linked to the high vacuum $\left(10^{-5}\right.$ to $\left.10^{-9} \mathrm{mbar}\right)$ in the interior of the mass spectrometer, c) electrons, photons as well as other particulates need to be stripped off the ion beam, as they cause signal instability and high background levels, and d) the ion beam needs to be accelerated and focused before it enters the mass separation and detector parts of the instrument. To achieve these tasks, the interface is equipped with two different cones that have small holes of around 0.8 to $1.2 \mathrm{~mm}$ and 0.4 to $0.8 \mathrm{~mm}$, respectively. The volume between the two cones is maintained at a vacuum of around one millibar by the interface pump that also removes most of the plasma and sample gases. After the second cone, a pumping system including several turbo pumps produce a high vacuum of $<10^{-7}$ mbar. Because of this strong difference in pressure between the interior of the instrument and the ICP region, the ions are sucked into the mass spectrometer. Here, an extraction lens selectively and actively accelerates positive ions away from the interface region into an ion optic system, which improves the transmission and ultimately the detection limit of low mass ions, as they tend to be at the edges of the ion beam and would otherwise be partly lost. The optic system then focuses, accelerates and shapes the ion beam onto the entrance slit, through which the ionized sample is passed on to the mass analyzer part of the MC-ICPMS. The main task of the mass analyzer part is to separate the ions based on their mass to charge ratio $(\mathrm{m} / \mathrm{z})$ with a magnet. However, a problematic aspect is that the plasma produces ions with a large range of kinetic energies, which would result in inferior mass separation. Therefore, an electrostatic analyzer (ESA) is placed before the magnet to filter the ions based on their kinetic energy. In some of the older generation MC-ICPMS instruments, collision cells have been used for this task instead of an ESA. In a collision cell, the ions collide with a gas (often Ar or He) and the energy transfer that occurs during these collisions reduces the energy spread of the ions. After the ions have been filtered for their kinetic energy, they pass the magnetic sector analyzer where they become separated by their $m / z$ ratio, and then they travel along different trajectories to several detectors that record the different isotopes of interest simultaneously.

The benefit of a MC-ICP-MS setup as described above is that it permits low background levels, high signal stability, flat peak tops and simultaneous analysis of several isotopes, which allows to analyze isotopic compositions of a large range of elements (masses from lithium to uranium) at relatively low concentrations (200 to $5 \mathrm{ng} / \mathrm{g}$ in solution). 


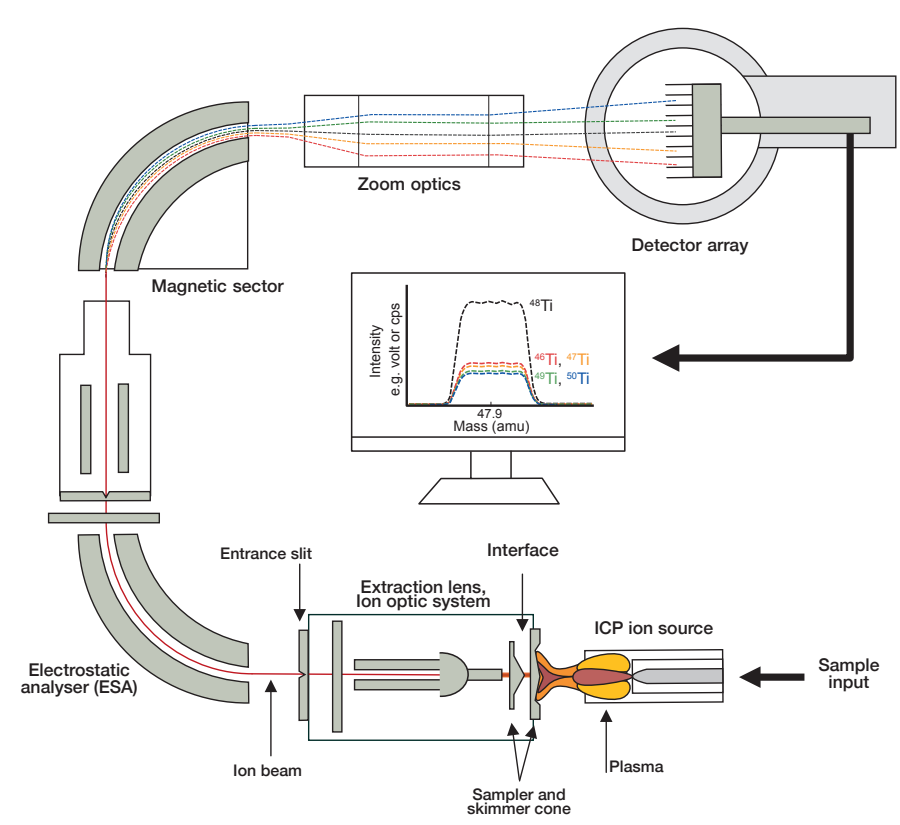

Fig. 1. Schematic and simplified setup of a MC-ICP-MS (modified after Schönbächler ${ }^{[14]}$ and Bazzano $\left.{ }^{[17]}\right)$. Such an instrument permits stable signals, flat peak tops, the simultaneous detection of several isotopes and allows for high precision isotope analysis of a large range of elements with masses from lithium to uranium.

\section{New Developments}

At present, the highest precision measurement of massdependent isotopic compositions is done by introducing a sample solution to the mass spectrometer that has been cleaned from its matrix elements with ion-exchange chromatography (see section Sample Preparation). However, using (femtosecond) laser ablation as sample introduction system has become more popular and the quality of the data increased significantly. ${ }^{[18,19]}$ Obviously, the benefit of in situ analysis of stable isotopes has the advantage of being able to measure the composition of the sample at high spatial resolution. The lower quality of the data when compared to MC-ICP-MS measurements in solution mode is mainly due to the complex matrix that is released by the laser ablation systems.

Matrix and (poly)atomic isobaric interferences are two common issues that prohibit the isotopic analysis of an element or hamper the quality of a MC-ICP-MS measurement. A prominent example is the impact of Ar-based interferences (added to the mass spectrometer at high concentration from the plasma) on ${ }^{40} \mathrm{~K}$. However, new MC-ICP-MS instruments that have been presented in the last years can now be equipped with a collision/reaction cell and a mass filter ${ }^{[20-22]}$ that help to circumvent these problems for the isotopic analysis of certain elements.

In the collision/reaction cell technology the ion beam reacts and collides with a gas or a mix of gases and the newly formed ions are afterwards accelerated again to a common kinetic energy. For example, adding $\mathrm{H}_{2}$ and $\mathrm{He}$ to the collision/reaction cell neutralizes charged polyatomic Ar species $\left(\mathrm{ArH}^{+}+\mathrm{H}_{2}\right.$ reacts to $\mathrm{H}_{3}^{+}+\mathrm{Ar}$ and $\mathrm{ArH}+\mathrm{H}_{2}^{+}$), which suppresses the Ar interference with $\mathrm{K}$ isotopes and therefore improves the quality of $\mathrm{K}$ isotope measurements. ${ }^{[23]}$ Another option is not to neutralize or modify the element responsible for the interferences, but the element of interest. For example, for the analysis of $\mathrm{Sr}$ or $\mathrm{Ti}$ isotopes these elements can react with $\mathrm{SF}_{6}$ or $\mathrm{O}_{2}$ gas, respectively, to form $\mathrm{SrF}^{+}$ and $\mathrm{TiO}^{+}$species that can then be analyzed for their isotopic ratios at masses with fewer isobaric interferences. ${ }^{[20]}$

A mass filter is a unit that allows only ions with a certain mass range to travel into the instrument. For example, for the analysis of $\mathrm{Sr}$ isotopes only isotopes with masses from 85 to 89 are allowed to move forward, while all the other ions will be deflected and removed from the system. A mass filter is therefore not only, but especially desirable for MC-ICP-MS measurements using a laser ablation system, as here purification of the sample prior to analysis is not possible. In the most recent MC-ICP-MS instruments, the mass filter is installed before the collision/reaction cell and these two units are placed in front of the extraction lens. Because the mass filter proceeds the collision/reaction cell it also improves the performance of the latter. This is because it strongly reduces the amount of material that enters the collision/reaction cell and therefore makes the interaction of the ion beam with the collision gases more effective. ${ }^{[20]}$ Furthermore, removing most of the unwanted isotopes prior to the collision/reaction cell limits the possible 'ion beam-gas' reactions that could occur, and therefore prohibits the production of other potential species with isobaric interferences on the masses that are analyzed. The combination of a mass filter and a collision/reaction cell might therefore be a key step in making MC-ICP-MS suitable for more robust and accurate in situ analysis of isotope ratios.

\section{Best Practice for High-precision Measurement of Mass-dependent Isotope Fractionation Sample Preparation}

Usually, samples are introduced as solution into an MC-ICPMS for analysis (apart from in situ analysis by laser ablation, which is, however, beyond the scope of this paper). This requires digestion of solid samples. Most often, the sample is brought into solution by applying different steps of acid digestion in a closed Teflon vessel on a hot plate. ${ }^{[24]}$ The exact protocol strongly depends on the sample material and the element that is analyzed and might also include sample digestion in a microwave (e.g. for organic-rich material) or under high pressure in a Parr ${ }^{\circledR}$ bomb (e.g. for highly resistant minerals like zircon and chromite). During acid digestion, secondary phases might precipitate that need further treatment. For instance, digestion of silicates with hydrofluoric acid (HF) often causes formation of secondary fluorides. For certain elements, incomplete sample digestion may bias the isotopic composition of the liquid, as either the incomplete digestion of the primary sample or the precipitation of secondary phases cause isotope fractionation. ${ }^{[25]}$ Therefore, often several digestion steps are necessary and sometimes the use of boric $(\mathrm{HBr})$ or perchloric acid $\left(\mathrm{HClO}_{4}\right)$ is indispensable for a complete digestion of fluoride precipitates or organic material. For rock powders or mineral samples, an alternative to direct acid digestion is to first melt the sample into a glass pellet, which then more easily dissolves in acid. In order to lower the melting point of the sample, it is first mixed with a flux (e.g. $\mathrm{NaOH}$ or $\mathrm{LiBO}_{2}$; see $\mathrm{Hu}$ and $\left.\mathrm{Qi}^{\mathrm{i}}{ }^{24]}\right)$. However, when using flux fusion it must be ensured that the element of interest does not evaporate during this processes (which could induce unwanted isotope fractionation).

Even more important, flux reagents often contain impurities in considerable amounts. When these impurities are mixed with the sample, they add to the so-called blank, i.e. contributions of the element of interest other than from the sample. The impact of the blank on the analyzed isotope ratio not only depends on the blank amount, but also on its difference in the isotopic composition from the sample ( $\Delta$ Sample-Blank). For instance, as little as $2.5 \%$ of blank contribution with a $\Delta$ Sample-Blank of $2.0 \%$ induces a shift of $0.05 \%$ on the isotope ratio, which is outside of a regularly achieved intermediate measurement precision for isotope ratios of trace elements (Fig. 2). Good control on and minimizing of the blank levels is thus essential for accurate high-precision isotope ratio measurements. Therefore, all reagents used during digestion and further sample preparation have to be of high purity. In practice, flux fusion digestion is avoided if possible and all acids are purified by (double) sub-boiling distillation. 


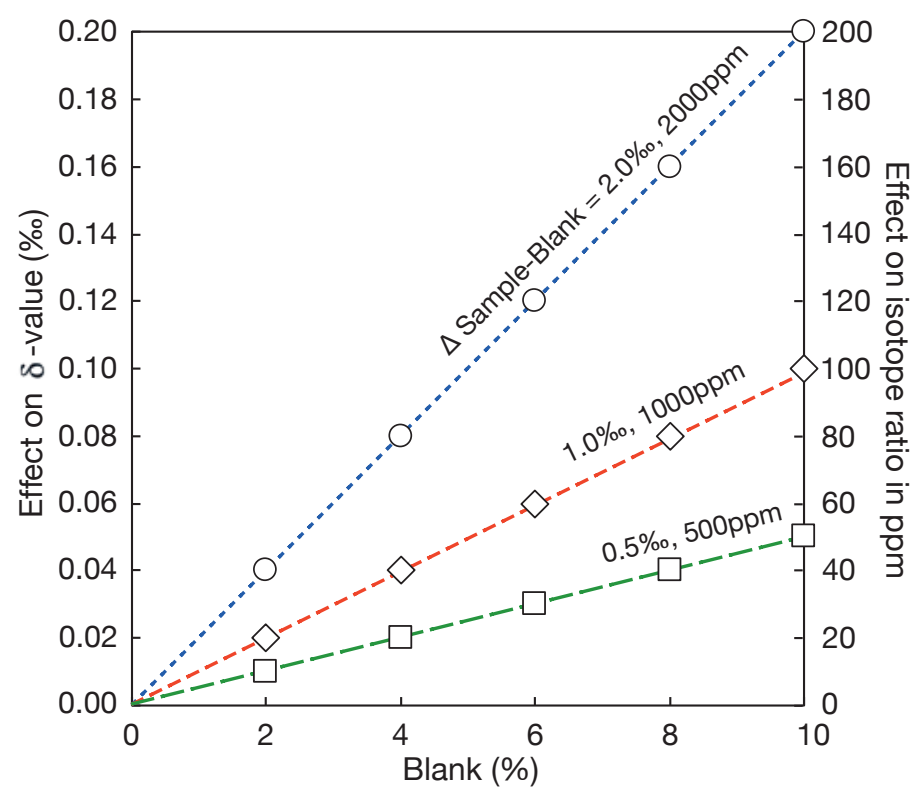

Fig. 2. Calculated impact of a blank on the isotopic composition of a sample ( $\delta$-value on left axis; in ppm on the right axis). Scenarios for three blanks are presented that have an isotopic offset of $0.5 \%, 1.0 \%$ o and $2.0 \%$ relative to the sample.

After digestion of the sample, the element of interest needs to be purified from the sample matrix (i.e. all elements other than that of interest). This is needed because isobaric interferences from a single element (e.g. ${ }^{48} \mathrm{Ca}$ on $\left.{ }^{48} \mathrm{Ti}\right)$ or polyatomic species (e.g. ${ }^{40} \mathrm{Ar}^{56} \mathrm{Fe}$ on ${ }^{96} \mathrm{Mo}$ ), but also pollution of the sample solution with matrix can result in the measurement of inaccurate isotope ratios. Sample purification is done by ion-exchange chromatography, or often simply called column chemistry. A column chemistry consists of a stationary (often a resin) and a mobile phase. At the beginning, the sample is dissolved in the mobile phase and mixed at one point with the stationary phase. The different species of the sample then interact between the mobile and the stationary phase, based on their partition coefficient towards each phase, ${ }^{[26]}$ meaning that certain species will remain in solution (mobile phase) and travel through the column, while others are preferentially retained by the resin (stationary phase). These days, a large range of different ion-exchange resins exists together with published partition coefficients for many elements and various acids and acid concentrations. ${ }^{[26,27]}$ It is therefore possible to produce high purity solutions of almost all elements at a reasonable work load. An example of an ion-exchange chromatography strategy using a TODGA resin from Eichrom ${ }^{\circledR}$ to preconcentrate $\mathrm{Ti}$ and $\mathrm{Zr}+$ Hf from its matrix is shown in Fig. 3. Here, Ti is eluted from the column together with $\mathrm{Nb}$, Mo and $\mathrm{W}$. For Ti isotope analysis, a second column that further purifies Ti from these elements is therefore required. ${ }^{28,29]}$

It has to be kept in mind that the ion-exchange chromatographic separation is a main contributor to the blank, originating from the acids and impure resins as well as from the users themselves. Resins should therefore always be cleaned prior to the ionexchange chromatography procedure and the user should pay close attention to avoid (cross-)contamination of their samples.

Another pitfall during this sample preparation step is that isotopes can be artificially fractionated on the column due to the different partition coefficient of different isotopes between the liquid and the resin. For instance, significant fractionation of $\mathrm{Cu}$ and $\mathrm{Zn}$ isotopes was found during column chemistry. ${ }^{[30]}$ While the lighter $\mathrm{Cu}$ and $\mathrm{Zn}$ isotopes remained longer in the resin bed, the heavier isotopes were eluted first. Incomplete collection of the sample's element of interest would thus alter its isotopic composition towards higher $\delta^{65 / 63} \mathrm{Cu}$ or $\delta^{66 / 64} \mathrm{Zn}$ values, leading to wrong isotope data. Therefore, one needs to ensure a quasi-complete recovery of the element of interest from the ion-exchange column. Alternatively, a so-called double spike can correct for the artificial fractionation. More details are given in the section Data Reduction.

\section{Instrument Setup and Measurement Routine}

After the ion-exchange chromatography, the sample is dried and redissolved in a weak acid (e.g. $0.05 \mathrm{M}$ nitric acid $\left(\mathrm{HNO}_{3}\right)$; termed here 'ICP solution'). For some elements, such as $\mathrm{Zr}$ and Ti, traces of hydrofluoric acid (HF) are added to increase their stability in solution. Hydrochloric acid $(\mathrm{HCl})$ is usually avoided as $\mathrm{Cl}^{-}$anions are highly reactive and may form additional unwanted polyatomic interferences. The sample solution is then introduced to the mass spectrometer as an aerosol, which is produced by a nebulizer. The nebulizer is either attached to a (glass) spray chamber, in which large aerosol droplets are removed, or to a desolvator, which dries the sample aerosol before reaching the plasma. A 'dry' plasma (desolvator) has a higher ionization efficiency than a 'wet' plasma

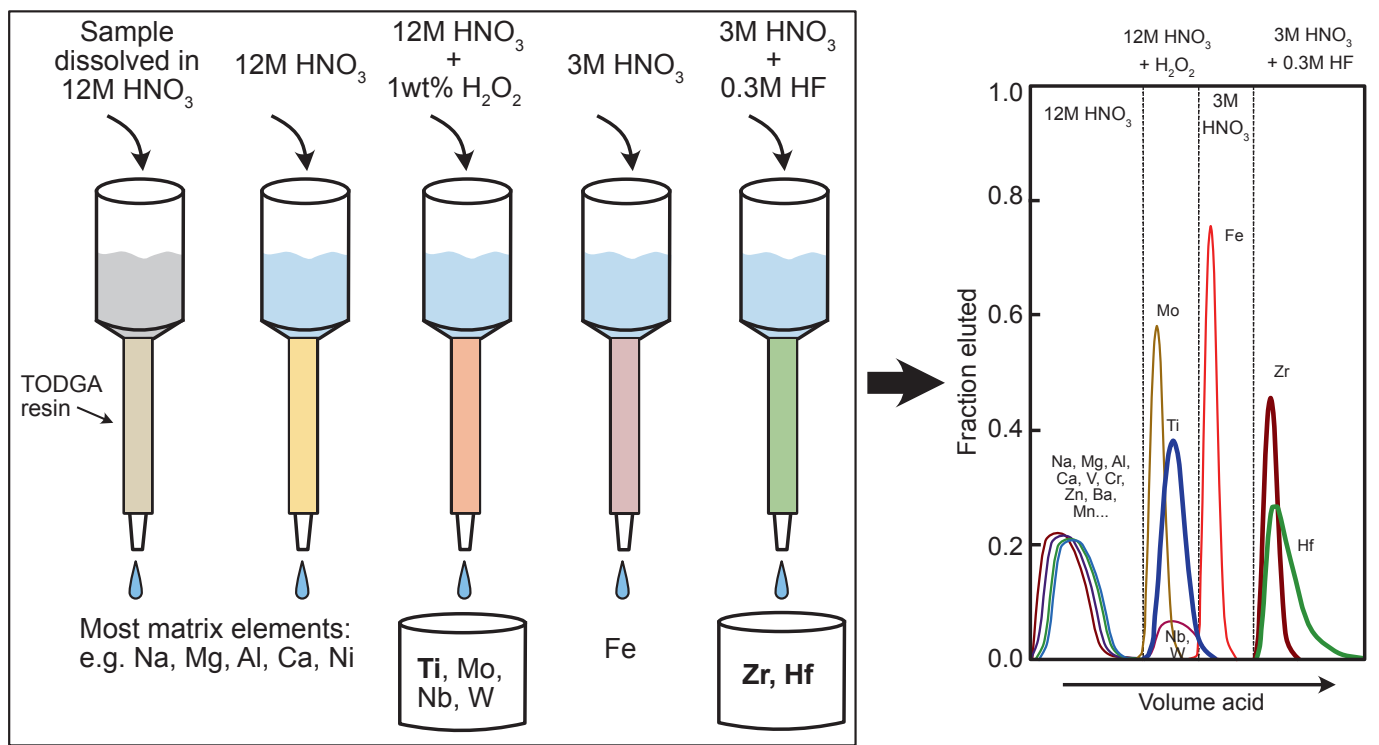

Fig. 3. Example of a ionexchange chromatography setup to isolate $\mathrm{Ti}, \mathrm{Zr}$ and $\mathrm{Hf}$ from their matrix using a TODGA resin from Eichrom ${ }^{\circledR}$. A second, different chromatography is needed to further purify Ti prior to measurement in MC-ICPMS. Proper purification of the element of interest is essential for accurate measurements, as remaining matrix elements may disturb isotope analysis through isobaric interferences and nonspectral matrix effects. 
(spray chamber) and therefore higher signals can be achieved. The best sample introduction system depends on the element to be analyzed and in what quantity it is available. Generally, sample introduction systems with a lower yield (i.e. spray chamber) produce a more stable signal and exhibit lower instrumentally induced isotope fractionation compared to those that allow for higher intensities (desolvator). For abundant elements, such as Fe, a spray chamber is often the best choice. However, to achieve high enough signal intensities for analysis of trace element isotopes, a desolvation system is often indispensable. For some elements, a completely different introduction system is needed. Selenium, for instance, cannot be efficiently introduced into the plasma with the described systems. Here, a hydride generator is necessary for sample introduction. ${ }^{[31]}$

Similarly, the setup of the two cones is variable and can be optimized for different elements. Cones with a smaller orifice are less sensitive and result in lower signals but produce less polyatomic interferences compared to more sensitive ones. The use of a pair of high sensitivity cones is normally the method of choice for elements that anyway require to be measured at high or medium resolution to avoid polyatomic interferences, as for example $\mathrm{Fe}$, $\mathrm{Ti}$ and $\mathrm{Cr}$ (Fig. 4). All of these elements have at least one isotope for which gas-based polyatomic interferences cannot be avoided in a MC-ICP-MS (i.e. ArO on Fe isotopes, ArN on Ti and $\mathrm{Cr}$ isotope). In case some of the expected interferences are difficult to see, one can also evaluate the position at which the polyatomic interferences start to impact the isotope signal of the element of choice by measuring a background or cleaning solution.

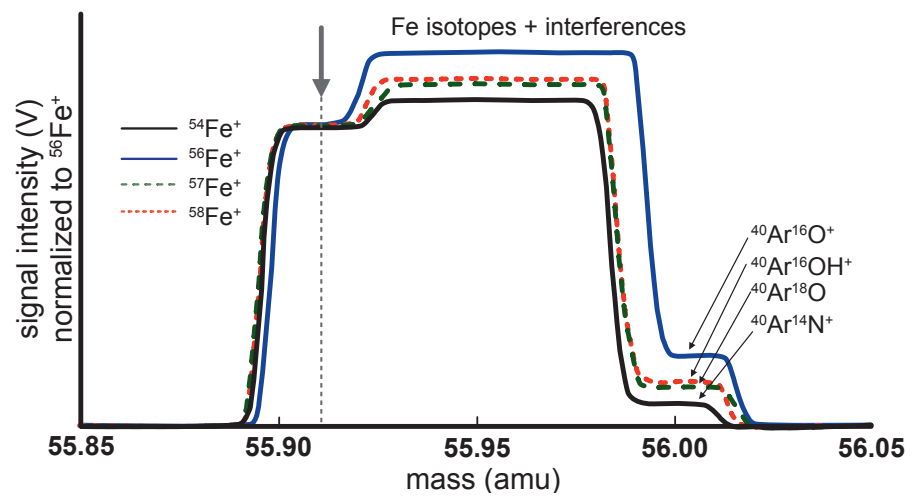

Fig. 4. Modified figure after Weyer and Schwieters. ${ }^{[32]}$ Peak scan over $\mathrm{Fe}$ isotope masses with a high enough resolution power to obtain a $54,56,57,58 \mathrm{Fe}$ isotope plateau free of unavoidable polyatomic interferences (ArO, ArOH and ArN). The axial magnet mass for an isotope measurement in this example is set at the interference free plateau at the low mass side of the peak, approximately indicated with the grey arrow and grey dashed line.

High-precision isotope analysis by MC-ICP-MS include the measurement of background, reference materials, secondary standards and the samples. The measurement with a MC-ICP-MS induces a large isotope fractionation, ${ }^{[13]}$ for which the obtained data needs to be corrected (see next section Data Reduction). Reference materials are solutions with known isotopic compositions that can be used to calibrate the instrument and to correct for the offset between the measured and defined isotopic composition of the solution. For non-traditional isotope measurements, the used reference material is often identical to the one in Eqn. (1), which translates to a defined $\delta$-value of $0 \%$. However, this is not a prerequisite and also reference materials with a $\delta$-value $\neq 0 \%$ can be used. For the measurements, the reference material should be dissolved in the ICP solution. A secondary standard is a material with a known isotope ratio that mimics the sample in its physical properties and chemical composition. The secondary standard is treated identically to a sample and thus undergoes the complete sample preparation and column chemistry procedure. A background consists of a measurement of the pure ICP solution (also called 'on-peak-zero') and is used to monitor and, if necessary, correct for signal contributions by the acid and plasma gases and by memory effects of the mass spectrometer on the different analyzed isotopes.

A measurement sequence usually consists of alternating analyses of reference material and sample. An on-peak-zero background can be measured before each analysis, sometimes it is however sufficient to do a background analysis before and after a block of around 10 samples. For quality control of the data, at least one secondary standard should be measured in each block. The best results are achieved if the reference materials and samples are measured at equal concentrations and signal intensities (ideally within $\pm 5 \%$ ). That way, background contributions from the detectors (electronic noise) and from the acid are the same for all solutions and are cancelled out.

\section{Data Reduction}

The transformation of the raw data into accurate isotope ratios includes normally the following steps: (i) background correction, (ii) correction of isobaric interferences, (iii) correction of isotope fractionation that is induced during the measurement in the MC-ICPMS (instrumental mass bias correction), and (iv) offset correction. Most of these corrections are rather straightforward and an example on how to perform a data reduction for $\mathrm{Ti}$ isotopes is given in Appendix A in the Supplementary Information. However, one of the peculiarities of MC-ICP-MS is that the measurement induces a large instrumental mass bias. ${ }^{[13]}$ This phenomenon is not fully explained, but next to the isotope fractionation that occurs during the sample introduction with a spray chamber or a desolvator, a large part of the isotope mass bias is likely related to the preferential extraction and transmission of heavier ions by the ion optic system. This instrumentally induced isotope fractionation ranges from around $12 \%$ per atomic mass unit (amu) for boron, to around 1\%/amu for uranium. ${ }^{[13]}$ This means that the instrumental isotope fractionation biases the measured isotope ratio around 200 to 2000 times more than the precision we aim for ( 1 to $10 \%$ compared to $\pm 0.03 \%$ o per amu; see Fig. 5). To obtain accurate and precise isotope ratios it is therefore essential to accurately correct for the instrumentally induced isotope fractionation. We will address here the three most often used strategies: (a) standard-sample bracketing, (b) elementdoping with standard bracketing and the (c) the double spike method.

All of the corrections for the instrumental mass bias use the exponential fractionation law, as several studies have shown that it adequately describes the isotope fractionation in an MC-ICPMS. ${ }^{[30]}$ We therefore start with relating the measured and true isotope ratios through:

$$
r^{i / j}=R^{i / j} \cdot\left(m_{i} / m_{j}\right)^{\beta}
$$

with $r$ being the measured ratio of isotopes $i$ and $j, R$ being the true ratio, $m$ being the masses of the isotopes and $\beta$ is a free parameter that describes the instrumental mass fractionation. ${ }^{[33]}$ For the measurement of radiogenic isotope signatures, one uses the same exponential fractionation law to do an internal normalization, where one isotope ratio is normalized to a fixed value. For example, for calculating the radiogenic ${ }^{87} \mathrm{Sr} /{ }^{86} \mathrm{Sr}$ isotopic composition, the $\beta$-value of the measurement is estimated using a 


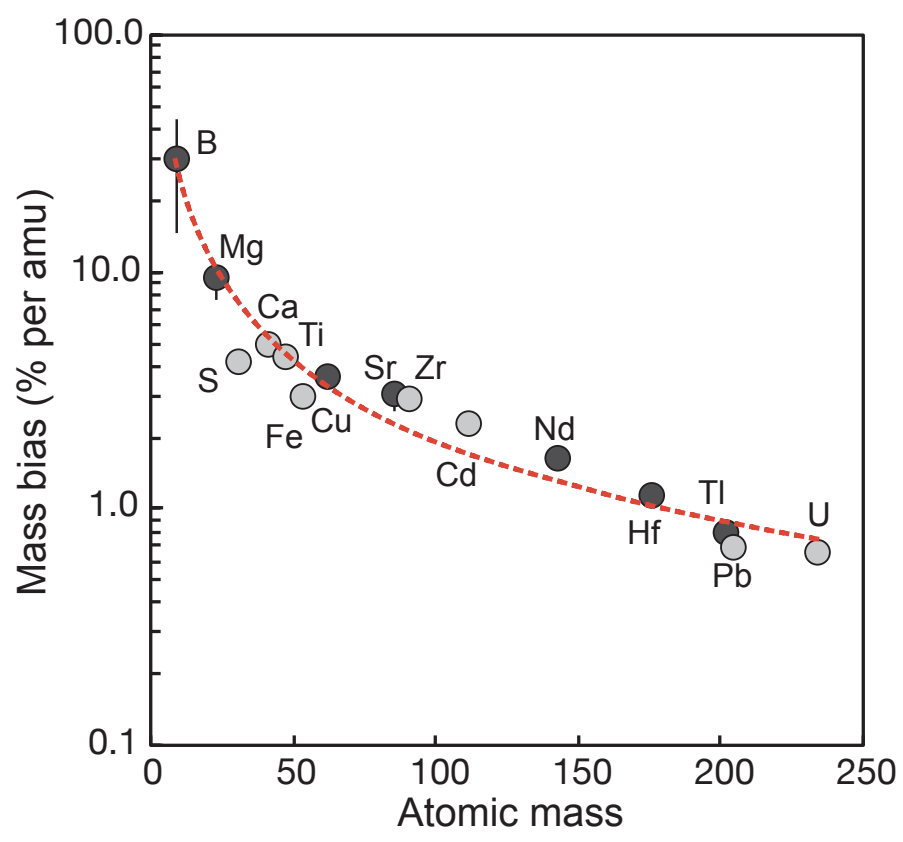

Fig. 5. Measured instrumental mass bias in a MC-ICP-MS in percent (\%) for different elements. Redrawn from Rehkämper et al., ${ }^{[13]}$ with $\mathrm{S}$ and $\mathrm{Ti}$ data added from this study. Elements shown with dark grey points were measured in different types of MC-ICP-MS ${ }^{[13]}$ and the thin bar illustrates the range of obtained data.

$R_{S r}^{86 / 88}$ of 0.1194 in Eqn. (2), and this $\beta$-value is then used to correct the measured ${ }^{87} \mathrm{Sr} /{ }^{86} \mathrm{Sr}$ ratio. However, by doing so, one not only eliminates the instrumental mass fractionation, but also the natural one, and for mass-dependent isotope studies the latter signature is the one to be determined. The three strategies on how to correct for the instrumental isotope fractionation for massdependent isotope studies therefore employ external corrections or a mix between external and internal corrections. For MC-ICPMS an external correction (i.e. the sample is not used for estimating the $\beta$-value) is made possible because the instrumental mass fractionation is relatively stable over a timespan which allows the analysis of both sample and reference material.

To correct for instrumental mass bias with the standard-sample bracketing technique, a sample is bracketed by two reference materials in a sequence of 'STD1 - SMP - STD2' (STD = standard/reference material, SMP = sample). Here, the $\beta$-value is calculated for each analysis of the reference material, which has a defined $R^{i / j}$. The measurement of the sample is then corrected following $\beta_{\text {Sample }}=0.5 \cdot \beta_{S T D 1}+0.5 \cdot \beta_{S T D 2}$. For Fe isotopes for example, one uses the reference material IRMM-014 with a $R_{F e}^{56 / 54}$ of 15.6986 in Eqn. (2) to calculate the $\beta$-values of the two standards, and the Fe isotopic composition of the sample can then be reported as $\delta^{56 / 54} \mathrm{Fe}$ with IRMM-014 as reference material in Eqn. (1). ${ }^{[33]}$ If the reference material used during analysis is the same as the one defining the $\delta$-scale, the mass bias uncorrected isotope ratios can directly be used for calculating the $\delta$-value:

$$
\delta^{\mathrm{i} / \mathrm{j}} \mathrm{A}(\%)=\left(\frac{R_{\text {Sample }}^{i / j}}{0.5 \cdot R_{\text {STD1 }}^{i / j}+0.5 \cdot R_{\text {STD2 }}^{i / j}}-1\right) \cdot 1000
$$

This has the advantage that the true isotope ratio of the reference material does not have to be known, as is the case for many non-traditional isotope systems. ${ }^{[34]}$
Bracketing your sample with standards has several advantages and is essential for high-precision isotope measurements. Firstly, the exponential law describes instrumental mass fractionation adequately, but not perfectly. The small difference between the calculated and the true mass bias is however similar for the reference material and the sample, and the standard bracketing therefore corrects also for this issue. ${ }^{[33]}$ Secondly, with standard-sample bracketing, a potential machine drift in the measured isotope ratios is automatically recorded at high temporal resolution. Such a drift during a measurement day can, for example, be induced by temperature fluctuations in the laboratory or by memory effects. Furthermore, the better the signal intensities of the recorded isotopes between the reference material and the sample match, the better these corrections work. Thus, for high precision isotope measurements, the signal intensity between reference material and sample should not be bigger than $\pm 5 \%$. A downside of the standardsample bracketing method is that $50 \%$ of the measurement time is used for the analysis of standards. However, as most instruments are nowadays equipped with an autosampler and measurements can run overnight, this is no longer a big disadvantage.

One bracketing cycle consisting of two reference materials and one sample measurement takes about 15-20 minutes. Within this time interval, a linear change in the instrumental mass bias must be assumed, as Eqn. (3) linearly interpolates between the two measured isotope ratios of the reference material. Often enough fluctuations in the mass bias, however, occur on a shorter time scale and the overall drift is sometimes not linear. In this case, the measurement solutions can be doped with another element that has a defined isotopic composition and that has a similar mass as the element of interest. Examples of element doping are $\mathrm{Ni}$ or $\mathrm{Cu}$-doping for $\mathrm{Fe}$ isotope measurements ${ }^{[33]}$ and $\mathrm{Mg}$-doping for $\mathrm{Si}$ isotope measurements. ${ }^{[35]}$ The $\beta$-value is then calculated using Eqn. (2) for the dopant and the mass bias corrected isotope ratios are used to calculate the $\delta$-value:

$$
\delta^{\mathrm{i} / \mathrm{j}} \mathrm{A}(\% \mathrm{o})=\left(\frac{{ }^{*} R_{\text {Sample }}^{i / j}}{0.5 \cdot{ }^{*} R_{\text {STD } 1}^{i / j}+0.5 \cdot{ }^{*} R_{\text {STD } 2}^{i / j}}-1\right) \cdot 1000
$$

With $* R^{i / j}$ being the isotopic ratios corrected for mass bias with the $\beta$-value obtained from the dopant. For Eqn. (4), the reference material (STD1 and STD2) has to be identical to the reference material in Eqn. (1).

This technique has been developed because the instrumental mass bias of a MC-ICP-MS is to a first order a function of the mass of an element (see Fig. 5). It therefore assumes that two elements of similar mass (the dopant and element of interest) behave similar in the mass spectrometer and that both have therefore similar $\beta$-values. In reality, however, the $\beta$-values of the dopant and the element of interest are not sufficiently identical for high-precision isotope analysis. It is therefore critical to bracket the sample with a reference material and that both solutions contain the same level of dopant and same amount of the element of interest. Further drawbacks of this technique are that the detector array in an MC-ICP-MS is often not large enough that the element of interest and the dopant can be measured simultaneously and that the element used for doping needs to be completely removed during the ion-exchange chromatography. If not, then the added dopant and the leftover of that element after column chemistry mix and alter the isotopic composition of the dopant. In other words, the isotopic composition of the dopant in the sample and in the reference material will no longer be identical, leading to erroneous results. The element doping technique is therefore not always favored over the more simple standard-sample bracketing correction.

The use of a double spike is the most robust and accurate technique to correct for isotope fractionation during MC-ICPMS measurements. ${ }^{[36,37]}$ A pioneering study in 2001 introduced 
this technique to the non-traditional stable isotope community by showing (i) that with this technique one can accurately correct for mass bias induced by the column chemistry and the instrument and (ii) that it allows to measure the Mo isotopic composition of various kinds of natural samples with an intermediate measurement precision of below $\pm 0.04 \%$ o/amu (2SD). ${ }^{[38]}$ Since then, the double spike technique has become the 'gourmet's choice' for the analysis of non-traditional stable isotopes. ${ }^{[39]}$ The double spike is a tracer with two artificially enriched isotopes that is mixed with the sample and that is made of the same element as is analyzed. The rationale behind utilizing a double spike is that the fractionation that is induced on the sample during sample preparation and isotope measurements will be mirrored by the double spike. The benefit of the double spike technique is that it allows for a precise correction of instrumental mass bias as well as for isotope fractionation that is imparted from sample preparation. Therefore, if the double spike is added prior to the column chemistry, a virtually full recovery of the element of interest is no longer necessary during this sample preparation step. Ideally, the double spike is even added before the digestion of the sample. However due to economic reasons this is sometimes avoided, as for the analysis of major elements like e.g. Fe and Ti too much of double spike would be wasted. Furthermore, the double spike also corrects to a certain degree for non-isobaric matrix effects, caused by matrix elements still present in the sample solution, and it corrects temporal fluctuation of the instrumental mass bias during a single analysis. It therefore makes an isotope measurement much more robust and enhances its accuracy and intermediate measurement precision.

For the data reduction of an isotope measurement that uses a double spike we rewrite Eqn. (2) for a sample-double spike mixture:

$$
r^{i / j}=\left[\lambda \cdot R_{\text {Spike }}^{i / j}+(1-\lambda) \cdot R_{\text {Sample }}^{i / j}\right] \cdot\left(m_{i} / m_{j}\right)^{\beta}
$$

Be aware that, (i) $\lambda$ does not directly solve for the proportion of double spike in the mixture and (ii), that the $\beta$-value stands here for the isotope fraction imparted during the isotope measurement and sample preparation. The solution for $\lambda$ depends on the isotope that is used as denominator in Eqn. (5) but it is related to the proportion of double spike in the mixture through Eqn. (B.1) that is given in Appendix B (following the equation in Rudge et al., [36] see Supplementary Information). Eqn. (5) has three unknowns $\left(\lambda, R_{\text {Sample }}^{i / j}\right.$ and $\left.\beta\right)$ and therefore requires three equations with three different isotope ratios to be solved (e.g. for Ti isotopes; ${ }^{46} \mathrm{Ti} /{ }^{47} \mathrm{Ti}$, ${ }^{48} \mathrm{Ti} /{ }^{47} \mathrm{Ti}$ and $\left.{ }^{49} \mathrm{Ti} /{ }^{47} \mathrm{Ti}\right)$. However, a problem is that $R_{\text {sample }}^{i / j}$ will not be identical among the three equations and therefore needs to be substituted with a term that comprises an invariant variable. For this, we relate the isotope ratio of the sample to that of the reference material by a mass-dependent fractionation law (the exponential law is often assumed) and write

$R_{\text {Sample }}^{i / j}=R_{\text {Reference Material }}^{i / j} \cdot\left(m_{i} / m_{j}\right)^{\alpha}$,

which in combination with Eqn. (5) leads to:

$$
r^{i / j}=\left[\lambda \cdot R_{\text {Spike }}^{i / j}+(1-\lambda) \cdot R_{\text {Reference Material }}^{i / j} \cdot\left(m_{i} / m_{j}\right)^{\alpha}\right] \cdot\left(m_{i} / m_{j}\right)^{\beta}(6)
$$

with $\alpha$ describing the natural mass-dependent isotope fractionation. Here, we have three invariant unknowns $(\lambda, \beta$ and $\alpha)$ and the system can be solved with three equations. From this it also follows that a priori the double spike technique requires element systems with four or more isotopes, so that three equations with three independent isotope ratios can be written (see also Coath et al., ${ }^{[40]}$ describing a special case for a double spike reduction with only three isotopes needed). To solve such a set of three exponential equations programs like R-Project, MatLab or Mathematica can be used. Furthermore, the double spike technique requires a spike and a reference material with known isotopic composition. Here, the spike is calibrated such that analysis of the reference material results in $\delta$-values equaling 0 . We direct the reader to one of the reviews describing the procedure on how to perform these calibrations. ${ }^{[11]}$ In practice, however, average $\delta$-values of the reference material of an analytical session often deviate from 0 and this deviation can vary between sessions. Therefore, similar to the element doping technique, best results are achieved if the sample is bracketed with a reference material that has a similar sample to spike ratio and signal intensity. The sample is then again corrected for the offset between the measured and defined isotopic composition of the bracketing reference material.

With the simple standard bracketing method or the element doping method intermediate measurement precisions of below $\pm 0.03 \%$ /amu (2SD) can be achieved for elements that are present in high concentrations (e.g. Fe and $\mathrm{Si}$ in most rocks). However, to achieve a similar intermediate measurement precision for minor and trace elements such as $\mathrm{Ba}, \mathrm{Ti}, \mathrm{Cr}$ and $\mathrm{Mo}$, the use of a double spike is generally necessary.

\section{Testing Accuracy, Robustness and Precision of a Measurement}

After developing a method to analyze the mass-dependent isotopic composition of an element it should be tested for its accuracy and for how robust it corrects for isobaric interferences and non-spectral matrix effects caused by leftover of matrix elements. For this, it is suggested to perform several tests:

a) The single element reference solution that is used for sample bracketing should be run once through the ionexchange chemistry. If the double spike technique is used, the solution should be spiked prior to the column chemistry. The reference material solution that has been run through the column chemistry and the untreated solution need to exhibit an identical isotopic composition. If this is not the case it might be that the reference material or the double spike is contaminated with matrix, or that the column chemistry introduces an unwanted bias (blank contribution or isotope fractionation during sample preparation).

b) If more than two isotopes of an element can be measured and the element doping or the standard-sample bracketing technique is employed, the data must plot on a straight line (massdependent fractionation line) in a 3-isotope plot. Deviating data often point toward unresolved interferences. Similarly, if the double spike technique is used and more than four isotopes can be measured, data can be reduced with two different sets of equations that utilize distinct isotope ratios. For instance, $\mathrm{Zr}$ isotope data can be reduced using the isotopes with masses 90 91-92-96 or 90-91-94-96 and a double spike that is enriched in ${ }^{91} \mathrm{Zr}$ and ${ }^{96} \mathrm{Zr}$. The isotope ratios after data reduction for the different sets of equations should be identical.[42]

c) Doping tests should be performed, where different matrix elements are added in different amounts to the reference material. This way the amount of remaining matrix that can be tolerated in the sample solution without disturbing the measured isotope ratios can be estimated.

d) A similar test should be done by doping a reference material solution with elements that have direct isobaric interferences that need to be corrected for. Interference corrections are generally based on two assumptions that are only approximately correct, (i) the interfering element is present in the sample solution in its natural isotopic abundance and (ii) the $\beta$-values of the interfering element and the element 
of choice are identical (see Eqn. A.3). It is thus necessary to evaluate what amount of contamination the interference correction can accurately correct for.

Furthermore, in each set of samples analyzed, one or ideally several secondary standards should be added and treated identically to a sample. This is a necessary quality control for each sample set that is analyzed. Preferably, the secondary standards should mimic the samples in their chemical and physical properties. A secondary standard should also be available in large enough quantity, so that it can be distributed and used for interlaboratory comparison. Additionally, they allow to evaluate the intermediate measurement precision of an isotope measurement, which is often reported as the 2SD of the secondary standards that have been measured over a longer period of time ( $>$ several months). The analyses of secondary standards is therefore essential for the evaluation of the accuracy and precision of the used measurement technique.

\section{Summary}

New developments in the field of isotope analysis and MCICP-MS in the last 20 years made it possible to measure the massdependent isotopic compositions of small amounts of sample material precisely and accurately. Nowadays, measurement protocols exist on how to measure the isotope ratios of almost all elements from $\mathrm{Li}$ to $\mathrm{U}$ that have more than one stable isotope. The fact that isotopic systems of a large range of elements with very different chemical properties can be measured with a precision that allows to resolve subtle isotope fractionation effects opens new research avenues for science and industry.

We shortly summarized and introduced the most important parts on how to do high-precision isotope analysis of non-traditional stable isotopes, which includes the tracking of laboratory blank levels, performing ion-exchange chromatography and correcting for instrumental mass bias. Furthermore, several tests are suggested that allow to determine whether or not an analytical protocol yields accurate isotope ratios.

Recent developments made it possible to implement mass filters and collision/reaction cells with a MC-ICP-MS. It is envisaged that this will further boost the utilization of MC-ICPMS instruments, as especially laser ablation in situ analysis will benefit from this progress. As such, MC-ICP-MS might also develop as one of the techniques of choice for the measurement of high precision isotopic compositions at high spatial resolution.

\section{Acknowledgments}

NDG acknowledges funding through the Swiss National Science foundation grant 181172. We also thank the reviewer and editor for constructive comments that increased the quality of the manuscript.

\section{Supplementary Information}

Supplementary information is available for this article at https://www.chimia.ch/

Received: November 29, 2021

[1] J. L. Birck, Rev. Mineral. Geochemistry 2004, 55, 25, https://doi.org/10.2138/gsrmg.55.1.25.

[2] J. Blichert-Toft, F. Albarède, Earth Planet. Sci. Lett. 1997, 148, 243 , https://doi.org/10.1016/S0012-821X(97)00040-X.

[3] J. Hoefs, 'Stable Isotope Geochemistry', Springer International Publishing, Cham, 2018, https://doi.org/10.1007/978-3-319-78527-1.

[4] M. A. Katzenberg, Biol. Anthropol. Hum. Skelet. Second Ed. 2007, 411, https://doi.org/10.1002/9780470245842.ch13.

[5] E. Niederschlag, E. Pernicka, T. Seifert, M. Bartelheim, Archaeometry 2003 , 45, 61, https://doi.org/10.1111/1475-4754.00097.

[6] F.-Z. Teng, N. Dauphas, J. M. Watkins, Rev. Mineral. Geochemistry 2017, 82, 1, https://doi.org/10.2138/rmg.2017.82.1.

[7] H. Cai, J. Chen, Sci. Bull. 2016, 61, 116, https://doi.org/10.1007/s11434-015-0968-8.

[8] J. Farquhar, J. Savarino, S. Airieau, M. H. Thiemens, J. Geophys. Res. Planets 2001, 106, 32829, https://doi.org/10.1029/2000JE001437.
[9] F. Albarède, in 'Encyclopedia of Astrobiology', Springer Berlin Heidelberg, Berlin, Heidelberg, 2011, pp. 611, https://doi.org/10.1007/978-3-642-11274-4 597.

[10] E. a Schauble, Rev. Mineral. Geochemistry 2004, 55, 65 https://doi.org/10.2138/gsrmg.55.1.65.

[11] C. R. McKinney, J. M. McCrea, S. Epstein, H. A. Allen, H. C. Urey, Rev. Sci. Instrum. 1950, 21, 724, https://doi.org/10.1063/1.1745698.

[12] J. H. Reynolds, J. Verhoogen, Geochim. Cosmochim. Acta 1953, 3, 224 https://doi.org/10.1016/0016-7037(53)90041-6.

[13] M. Rehkämper, M. Schönbächler, C. H. Stirling, Geostand. Newsl. 2001, 25, 23, https://doi.org/10.1111/j.1751-908X.2001.tb00785.x.

[14] M. Schönbächler, in 'Encyclopedia of Geochemistry', Vol. 2, 2016, pp. 1, https://doi.org/10.1007/978-3-319-39193-9_111-1.

[15] M. Rehkämper, M. Schönbächler, R. Andreasen, Isot. Anal. Fundam. Appl. Using ICP-MS 2012, 275, https://doi.org/10.1002/9783527650484.ch10.

[16] R. Thomas, Spectrosc. (Santa Monica) 2003, 18, 42.

[17] A. Bazzano, Ph.D. Thesis University of Genoa, 2016.

[18] P. J. Le Roux, J. Anal. At. Spectrom. 2010, 25, 1033, https://doi.org/10.1039/b920341a.

[19] M. Oeser, S. Weyer, I. Horn, S. Schuth, Geostand. Geoanalytical Res. 2014, 38, 311, https://doi.org/10.1111/j.1751-908X.2014.00288.x.

[20] G. Craig, H. Wehrs, D. G. Bevan, M. Pfeifer, J. Lewis, C. D. Coath, T. Elliott, C. Huang, N. S. Lloyd, J. B. Schwieters, Anal. Chem. 2021, 93, 10519, https://doi.org/10.1021/acs.analchem.1c01475.

[21] Y. Zhao, P. S. Pereira, T. van de Flierdt, M. Rehkämper, in 'Goldschmidt2021, Virtual, 4-9 July', 2021.

[22] F. Moynier, Y. Hu, K. Wang, Y. Zhao, Y. Gérard, Z. Deng, J. Moureau, W. Li, J. I. Simon, F. Z. Teng, Chem. Geol. 2021, 571, https://doi.org/10.1016/j.chemgeo.2021.120144.

[23] W. Li, B. L. Beard, S. Li, J. Anal. At. Spectrom. 2016, 31, 1023, https://doi.org/10.1039/c5ja00487j.

[24] Z. Hu, L. Qi, Treatise Geochemistry Second Ed. 2013, 15, 87, https://doi.org/10.1016/B978-0-08-095975-7.01406-6.

[25] X. He, J. Ma, G. Wei, L. Zhang, Z. Wang, Q. Wang, J. Anal. At. Spectrom. 2020, 35, 100, https://doi.org/10.1039/c9ja00316a.

[26] M. Schönbächler, M. A. Fehr, Treatise Geochemistry Second Ed. 2013, 15 , 123, https://doi.org/10.1016/B978-0-08-095975-7.01408-X.

[27] A. Pourmand, N. Dauphas, Talanta 2010, 81, 741 https://doi.org/10.1016/j.talanta.2010.01.008.

[28] J. Zhang, N. Dauphas, A. M. Davis, A. Pourmand, J. Anal. At. Spectrom. 2011, 26, 2197, https://doi.org/10.1039/c1ja10181a.

[29] N. D. Greber, N. Dauphas, I. S. Puchtel, B. A. Hofmann, N. T. Arndt, Geochim. Cosmochim. Acta 2017, 213, 534, https://doi.org/10.1016/j.gca.2017.06.033.

[30] C. N. Maréchal, P. Télouk, F. Albarède, Chem. Geol. 1999, 156, 251, https://doi.org/10.1016/S0009-2541(98)00191-0.

[31] T. Kurzawa, S. König, J. Labidi, A. Yierpan, R. Schoenberg, Chem. Geol. 2017, 466, 219, https://doi.org/10.1016/j.chemgeo.2017.06.012.

[32] S. Weyer, J. B. Schwieters, Int. J. Mass Spectrom. 2003, 226, 355 , https://doi.org/10.1016/S1387-3806(03)00078-2.

[33] N. Dauphas, A. Pourmand, F. Z. Teng, Chem. Geol. 2009, 267, 175, https://doi.org/10.1016/j.chemgeo.2008.12.011.

[34] K. Moeller, R. Schoenberg, R. B. Pedersen, D. Weiss, S. Dong, Geostand. Geoanalytical Res. 2012, 36, 177, https://doi.org/10.1111/j.1751-908X.2011.00153.x.

[35] F. Poitrasson, Non-Traditional Stable Isot. 2017, 82, 289, https://doi.org/10.2138/rmg.2017.82.8.

[36] J. F. Rudge, B. C. Reynolds, B. Bourdon, Chem. Geol. 2009, 265, 420, https://doi.org/10.1016/j.chemgeo.2009.05.010.

[37] M. H. Dodson, J. Phys. E. 1969, 2, 490 , https://doi.org/10.1088/0022-3735/2/6/306.

[38] C. Siebert, T. F. Nägler, J. D. Kramers, Geochem. Geophys. Geosys. 2001, 2 , https://doi.org/10.1029/2000GC000124.

[39] T. F. Nägler, in 'Goldschmidt Conference, Davos', 2002, p. A545.

[40] C. D. Coath, T. Elliott, R. C. Hin, Chem. Geol. 2017, 451, 78, https://doi.org/10.1016/j.chemgeo.2016.12.025.

[41] M. Klaver, C. D. Coath, Geostand. Geoanalytical Res. 2019, 43, 5, https://doi.org/10.1111/ggr.12248.

[42] H. G. D. Tompkins, L. J. Zieman, M. Ibañez-Mejia, F. L. H. Tissot, J. Anal. At. Spectrom. 2020, 35, 1167, https://doi.org/10.1039/c9ja00315k.

\section{License and Terms}

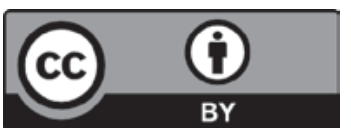

This is an Open Access article under the terms of the Creative Commons Attribution License CC BY 4.0. The material may not be used for commercial purposes.

The license is subject to the CHIMIA terms and conditions: (https://chimia.ch/chimia/about).

The definitive version of this article is the electronic one that can be found at https://doi.org/10.2533/chimia.2022.18 